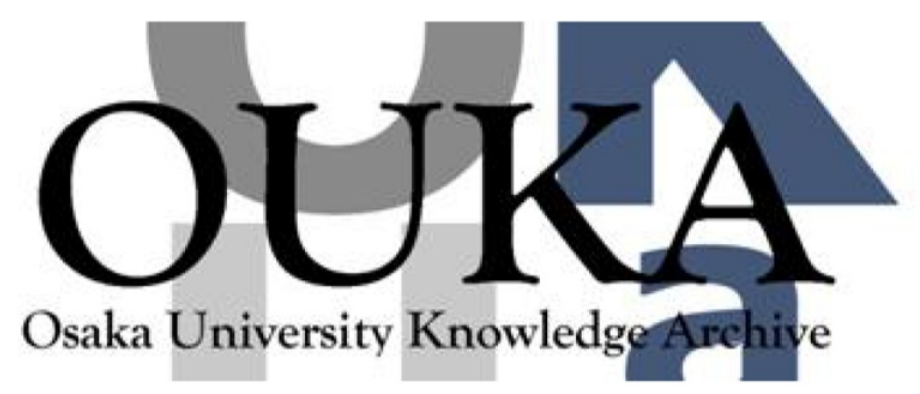

\begin{tabular}{|c|l|}
\hline Title & $\begin{array}{l}\text { Analysis of VHF-wideband electromagnet ic noises } \\
\text { from partial discharge using discrete wavelet } \\
\text { transform }\end{array}$ \\
\hline Author(s) & $\begin{array}{l}\text { Tungkanawanich, A.; Hamid, E. Y. ; Kawasaki, Z. - } \\
\text { I. et al. }\end{array}$ \\
\hline Citation & $\begin{array}{l}\text { Proceedings of the IEEE Power Engineering } \\
\text { Society Transmission and Distribution } \\
\text { Conference. 1 p. 263-p. 268 }\end{array}$ \\
\hline Issue Date & $2001-01$ \\
\hline oaire:version & VoR \\
\hline URL & $\begin{array}{l}\text { https://hdl. handle. net/11094/14057 } \\
\text { c2001 IEEE. Personal use of this material is } \\
\text { permitted. However, permission to } \\
\text { reprint/republish this material for advert is ing } \\
\text { or promotional purposes or for creat ing new } \\
\text { collective works for resale or redistribution } \\
\text { to servers or lists, or to reuse any } \\
\text { copyrighted component of this work in other } \\
\text { works must be obtained from the IEEE. . }\end{array}$ \\
\hline rights \\
\hline Note & \begin{tabular}{l} 
\\
\hline
\end{tabular} \\
\hline
\end{tabular}

Osaka University Knowledge Archive : OUKA

https://ir. Library. osaka-u. ac. jp/

Osaka University 


\title{
Analysis of VHF-wideband Electromagnetic Noises from Partial Discharge Using Discrete Wavelet Transform
}

\author{
A. Tungkanawanich, Student Member, IEEE E.Y. Hamid, Non-member \\ Z-I. Kawasaki, Member, IEEE K. Matsuura, Non-member \\ Graduate School of Electrical Engineering, Osaka University, Suita, Osaka 565-0871, JAPAN \\ E-mail: ampol@pels.pwr.eng.osaka-u.ac.jp
}

\begin{abstract}
In this paper, a new application of discrete wavelet transform to analyze the wide-band electromagnetic waves from partial discharge (PD) is introduced. The discrete wavelet transform has a special feature to perform the signal decomposition and reconstruction by multi-resolution analysis. Using this method, we can decompose the detected signal into various frequency sub-band signals, and then estimate the true shape of PD signal by mixing the appropriately-selected decomposed signals. The threshold processing of sub-band signals is applied to reduce the interference of broadcasting signals. It is shown that the proposed method is advantageous to obtain almost the true shape of PD signal, whose characteristics can be used for the purpose of PD classification. Experimental results obtained by applying high voltage on faulty insulators are used to show the effectiveness of the proposed method.
\end{abstract}

Keywords - partial discharge, electromagnetic waves, VHF-wideband detection, discrete wavelet transform, power distribution lines.

\section{INTRODUCTION}

$\mathrm{R}$ ECENTLY, there is a great increase in topics concerning electromagnetic (EM) environment influenced by HV equipment. The EM waves emitted from HV equipment may harm the human health and interfere the wireless communication systems as noises [1], [2]. One of the main sources of EM noises is the partial discharge (PD), which occurs when there are abnormal electric field stress conditions within insulation system. Therefore the detection, location and classification of PD are very important to enhance the reliability of power system and the electromagnetic environment [3], [4].

The EM radiation from $\mathrm{PD}$ normally exhibits the feature of transient wide-band signals in few hundred nanoseconds, with various intensities. In order to detect $\mathrm{PD}$, we utilize the VHF wide-band antenna to measure the electromagnetic signals from PD. The EM radiation from PD is generated by particles falling to lower energy states and shows the different feature depending on the kind and mechanism of PD source [5]. Unlike the narrow-band detection, the wide-band detection has an advantage to record nearly the true shape of an EM wave, which is useful information to classify PD.
Due to the local electromagnetic environment or the background noises, the shape of EM wave is distorted on their way from PD source to the measurement device. This makes the analysis of PD signal wrong if the emitted EM wave is not strong enough. The method to extract the true shape of PD signals requires an appropriate signalprocessing technique to be developed.

In this paper, we employ a discrete wavelet transform to analyze the wide-band signals. The discrete wavelet transform has a special feature to perform the signal decomposition and reconstruction by multi-resolution analysis. Using this method, we can decompose the detected signal into various frequency sub-band signals, and then estimate the true shape of PD signal by mixing the appropriatelyselected decomposed signals. Furthermore, the original and reconstructed signals are compared and analyzed by fast Fourier transform and continuous wavelet transform. It is shown that the proposed method is advantageous to obtain the true shape of PD signal, whose characteristics can be used for the purpose of PD classification. Experimental results obtained by applying high voltage on faulty insulators are used to show the effectiveness of the proposed method.

\section{EXPERIMENT}

The partial discharge from faulty insulators on distribution lines is concentrated. The inferior contact of two strain insulators as shown in Fig. 1 is used as a PD source in the experiment. The inferior contact was constructed by wrapping the insulated tape around a cotter pin (the pin used to combine two strain insulators together). The reason why we selected this kind of PD is that the EM noise sources from this PD are more than 80 [\%] of all sources [2]. In Japan, the $6.6 \mathrm{kV}$ power distribution systems are mostly adopted. For this system, the phase voltage is 3.81 $\mathrm{kV}$ so we applied $60 \mathrm{~Hz}$ ac high voltage $4 \mathrm{kV}$ upon a $P D$ source.

Figure 2 shows an experimental configuration. The experiment was done on the outdoor roof of a building where is plain and free of obstacles. The PD source was positioned at $d=17 \mathrm{~m}$ far from the VHF wide-band capacitive plate antenna. The EM signals received at antenna were passed through the band-pass filter $(25-250 \mathrm{MHz})$ and digital storage oscilloscope (DSO: Lecroy 9374L) by coaxial cable (type: 5D-2V, connector: BNC, length: $30 \mathrm{~m}$ ). The 


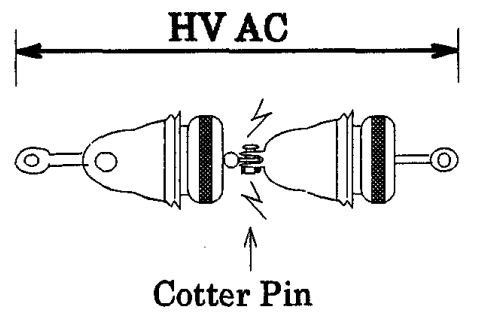

Fig. 1. A PD source used in the experiment (faulty contact of insulators)

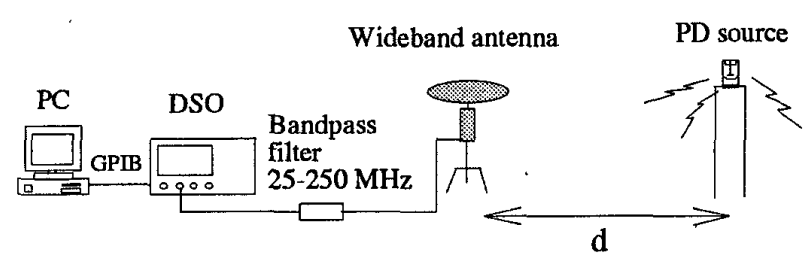

Fig. 2. An experimental configuration

EM waves were digitized at a sampling rate $500 \mathrm{MHz}$ by using a DSO controlled by a personal computer (PC) through GPIB board, with the resolution 8 bits. Each waveform, whose length of 1000 samples or $2 \mu \mathrm{s}$, is detected by adjusting the appropriate triggering level, with a trigger delay 30 [\%].

\section{ANALYSIS OF WIDE-BAND EM WAVES}

In this paper, three kinds of signal-processing techniques, which are fast Fourier transform, continuous wavelet transform and discrete wavelet transform, are utilized. The brief explanations of them are given as follows.

\section{A. Fast Fourier transform}

For many years, the fast Fourier transform (FFT) is the most popular algorithm to make a Fourier transform of a signal. FFT has a special feature for extracting the average spectral intensities of an entire signal fast and efficiently. However, the weak point of FFT is that the FFT provides the result expressed in only the frequency domain where the information of time domain would be lost.

\section{B. Continuous wavelet transform}

To overcome the limitation of Fourier transform, wavelet transform has been applied to perform time-frequency analysis in numerous problems [6], [7]. A continuous wavelet transform (CWT), one of the main algorithms of wavelet transform, is generally used to obtain the time-varying information of signal in detail. The CWT is the measurement of the similarity between a signal and a set of fast-decaying oscillating functions named wavelets. Each wavelet is constructed by compressing or dilating an original function called mother wavelet.

The CWT of signal $\mathrm{x}(\mathrm{t})$ with mother wavelet $\psi(\mathrm{t})$ is expressed as

$$
C W T(f, b)=\sqrt{2 \pi f} \int_{-\infty}^{\infty} \psi^{*}[2 \pi f(t-b)] x(t) d t
$$

where $f$ and $b$ is the frequency and time parameters, respectively, and $\left(^{*}\right)$ denotes a complex conjugate. An energy normalization factor $\sqrt{2 \pi f}$ is multiplied to keep the energy of the compressed wavelets unchanged from the original one. In this paper, one of the family of complex Gabor functions, which are mainly developed from Gaussian function, as shown in (2) is selected to be a mother wavelet.

$$
\psi(t)=e^{-\frac{t^{2}}{64}} e^{-i t}
$$

The CWT can provide the spectral intensities of any points on time-frequency space. Therefore, we can compare the result with FFT analysis. However, the disadvantages are the redundancy of the result and the time consuming due to the computation on the entire time-frequency space.

\section{Discrete wavelet transform}

Although the CWT can be used to represent the timefrequency analysis of a signal, its result is highly redundant especially in case the reconstruction of signal is concerned. This redundancy requires a great amount of processing time and memory. Unlike the CWT, the discrete wavelet transform (DWT) can reduce the computation time and storage memory, and still provide sufficient information for time-frequency analysis [8].

The DWT of a discrete signal $\mathrm{x}[\mathrm{n}]$ obtained by sampling frequency fs can be performed by first passing it through a half-band high-pass filter $g[n]$ and a low-pass filter $h[n]$. Note that the total number of samples $(N)$ should be the power of $2\left(\mathrm{~N}=2^{\mathrm{i}}\right.$, where $\mathrm{i}$ is an integer). After filtering, the every other sample (half of them) can be discarded according to Nyquist's rule, since the signal now has a maximum frequency of $\mathrm{fs} / 4$ instead of $\mathrm{fs} / 2$. This process constitutes one level of decomposition and can mathematically be expressed as

$$
\begin{aligned}
& y_{\text {high }}[k]=\sum_{n} x[n] \cdot g[2 k-n] \\
& y_{\text {low }}[k]=\sum_{n} x[n] \cdot h[2 k-n]
\end{aligned}
$$

where $y_{\text {high }}[k]$ and $y_{\text {low }}[k]$ are the outputs of the high-pass and low-pass filters, respectively, after sub-sampling by 2 . Note that two filters, $g[n]$ and $h[n]$, are categorized in a sort of the Quadrature Mirrors Filters (QMF) [8], and are related to each other by

$$
g[L-1-n]=(-1)^{\mathrm{n}} \cdot h[n]
$$

where, $\mathrm{L}$ is the filter length in number of points. Figure 3 shows the DWT decomposing algorithm. The decomposed signal has a half time resolution because the original signal is sub-sampled by 2 . On the contrary, it has a double frequency resolution because the frequency band now covers 


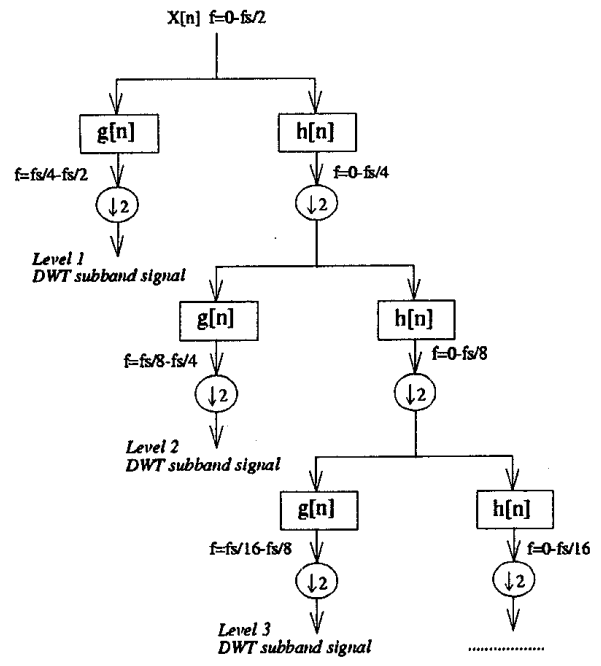

Fig. 3. DWT algorithm

source under the same condition but at the different time. The characteristics of both waveforms, roughly seen by eyes, are normally the same but the amplitudes are different. The amplitude of the former (Fig. 4 (b)) is little stronger than that of background noises, so its normalized spectral results, without any pre-processing procedures, are significantly influenced by noises. On the contrary, the amplitude of the latter (Fig. 4 (c)) is much higher than the level of background noises, so its normalized spectral results are mostly not influenced by noises.

From these results, the FFT and CWT analyses in Figs. 4 (b) and (c) are different to each other even the PD sources are the same. (Notice that the Fig. 4 (d) will be explained in a next section.) It is discussed that the classification of PD sources by considering the spectral characteristics of emitted EM waves, which was presented in [5], might not be efficient method if the amplitudes of signals are not high enough. The efficient method to extract the true shape of EM waveform from noises is required to be developed.

\section{B. DWT Analysis}

only half of the previous one. This procedure is called a $s u b$-band coding. The repetition of this procedure provides the further decomposed signals. The reconstruction of signal at each level can be simply performed by computing the following formula.

$$
x[n]=\sum_{k}\left(y_{\text {high }}[k] \cdot g[2 k-n]\right)+\left(y_{\text {low }}[k] \cdot h[2 k-n]\right)
$$

In this paper, we choose the coefficients of Daubechies-8 [9], to construct the low-pass and high-pass filters for signal analysis.

\section{RESULTS AND DISCUSSIONS}

\section{A. FFT and CWT Analyses}

Firstly, the EM environment (background noises) at experimental site is considered. Figure $\mathbf{4}$ (a) shows the background noises and their FFT and CWT analyses. Note that, in order to standardly compare the results, the normalized values of spectral intensities are used to express the FFT and CWT analyses. The frequency peaks in the Fourier spectrum are thought to be the signals from radio and television broadcastings. In Japan, the frequency range $76.1 \sim 89.9 \mathrm{MHz}$ is accordant with $\mathrm{FM}$ radio signals. The peaks in the frequency range $90 \sim 108 \mathrm{MHz}$ are supposed to be the signals from TV broadcasting of channels $1 \sim 3$. The peaks in $170 \sim 222 \mathrm{MHz}$ are expected to be the signals from TV channels 4 12 [2]. Moreover, from the same Fig., the wavelet spectrum exhibits the continuous occurrence of these broadcasting frequencies. These results confirm that the wide-band VHF signals were correctly measured by the antenna used in this experiment.

Secondly, the EM waves emitted from faulty insulators (faulty contact) are analyzed. Figures 4 (b) and (c) show two examples of EM waves and their FFT and CWT analyses. Both EM waves were obtained from the same PD

Because the number of samples in each waveform equals 1000 , which is not the power of 2, two 12-number arrays whose all values equal 0 were added at both the head and bottom of a waveform array. Therefore, we can get the 1024 samples of each waveform for DWT analysis. The DWT results of EM waves in Figs. 4 (a) and (b) in 5 levels of decomposition are illustrated in Figs. 5 (a) and (b), respectively. The analyzed frequency band is noted at each level and the amplitude of the decomposed signal shows the intensities of the spectrum of its own frequency band.

Considering the analysis of background noises (Fig. 5 (a)), the amplitude of signal in level-2 $(\mathrm{f}=62.5 \sim 125 \mathrm{MHz})$ is highest, that is accordant to the FFT result. Moreover, because these noises are from the broadcasting signals, the sub-band signals of all levels are almost stationary, which is accordant to the CWT result.

In case of EM wave from faulty insulators (Fig. 5 (b)), there are the remarkable peaks occurring in all levels except level-5, during the time when the pulse occurs in the original signal (around 500-700 ns). It can be postulated that the EM wave from PD is a wide-band signal whose frequency range covers most levels' frequency bands. The DWT has a special feature that can reconstruct a signal using the sub-band signals from its analysis. The noise reduction can be easily done by discarding the frequency bands where noises are included. Unfortunately, as can be seen from Fig. 5 (b), the background noises have main components in the level-2, where the PD signal also exists. The elimination of entire signal of this level makes the reconstructed signal lose its significant characteristics.

In order to obtain the reconstructed signal whose shape is nearly the true shape of PD signal, the threshold processing of sub-band signals is applied. The threshold value of each level is set at the maximum absolute value of sub-band signal of background noises in its own level. If the value of sub-band signal is less than the threshold value, it will 


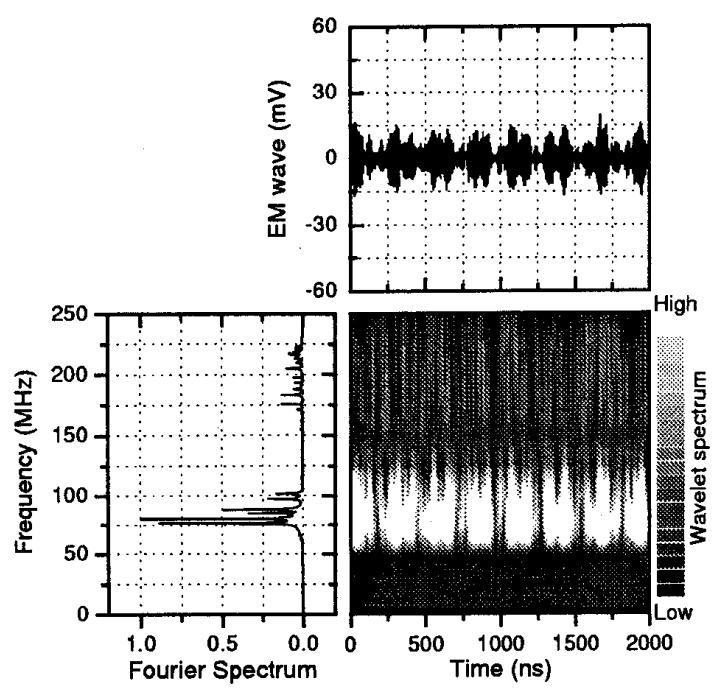

(a) background noises

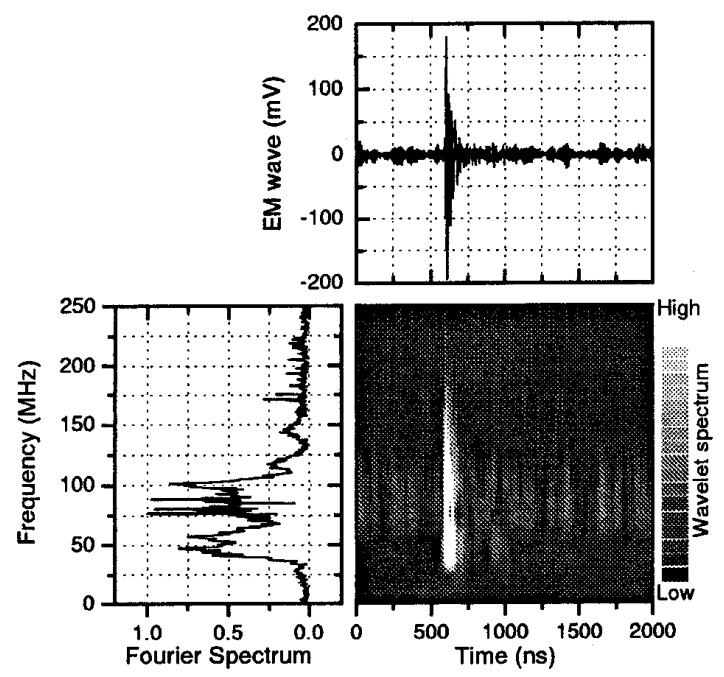

(c) faulty insulators No.2

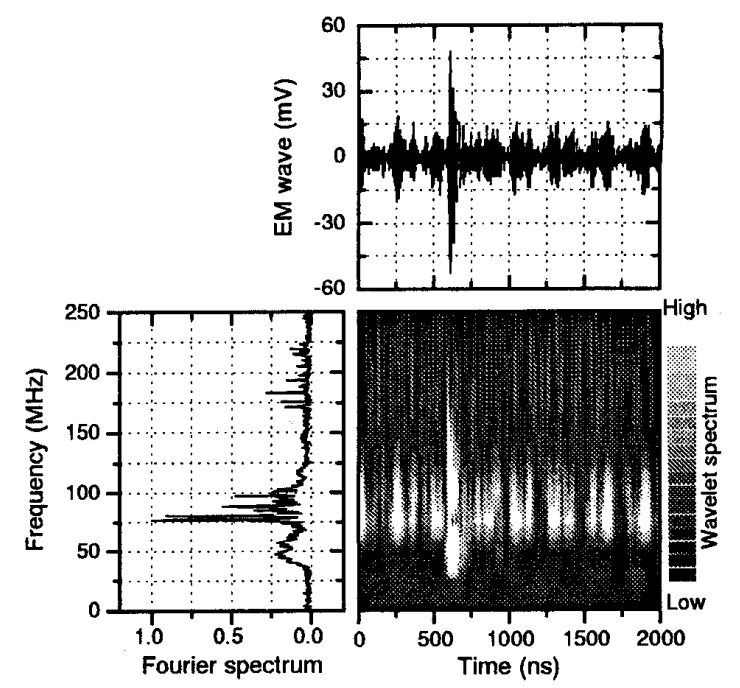

(b) faulty insulators No.1

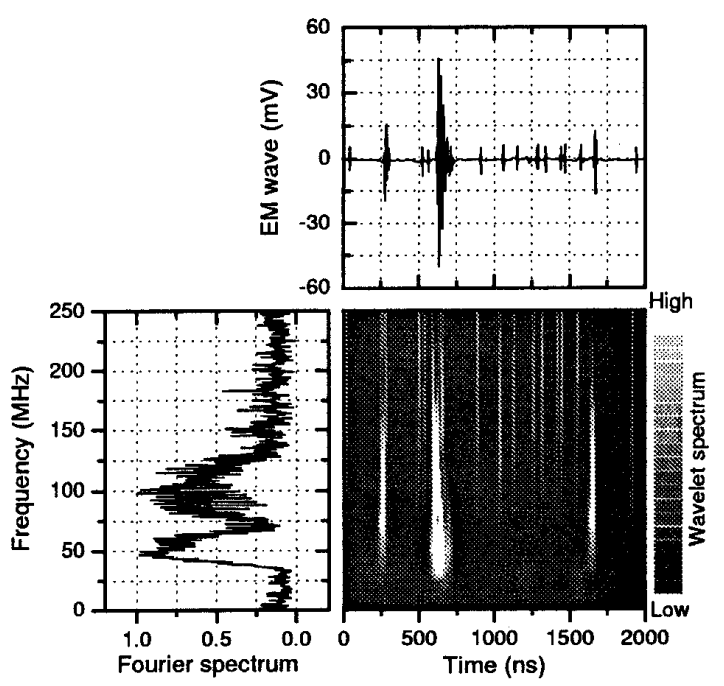

(d) the reconstructed signal of (b)

Fig. 4. The FFT and CWT results of wide-band EM waves

be changed to 0 , else, it will be unchanged. After this process has been applied, the high values of sub-band signals, which normally contain the information of PD signal, will be maintained. On the other hand, the low values, which are mainly accordant to the noises, will be eliminated. Figure 5 (c) shows the decomposed sub-band signals of Fig. 5 (b) after threshold processing taken.

Figures 6 shows the original and reconstructed signals, and the error signal, obtained by using the new sub-band signals of Fig. 5 (c). It is clearly seen that the EM pulse in reconstructed signal is nearly the same as the original one. The error signal also resembles the background noises in Fig. 4 (a).

To investigate the spectral contents in the reconstructed signal, we again applied the FFT and CWT analyses and its results are shown in Fig. 4 (d). Comparing with the results in Fig. 4 (b), the FFT and CWT results are different and their characteristics become to resemble those in Fig. 4 (c), which are little influenced by noises.

\section{CONCLUSIONS}

A new application of discrete wavelet transform for analyzing wide-band electromagnetic signals from partial discharge (PD) has been introduced. The discrete wavelet transform decomposes a signal into various sub-band levels, which provides us roughly the characteristics of spectra in their frequency bands without redundancy. Moreover, The reconstruction of a signal by using discrete wavelet transform result can extract almost the true shape of PD signal even in noisy condition. It has been shown that the 


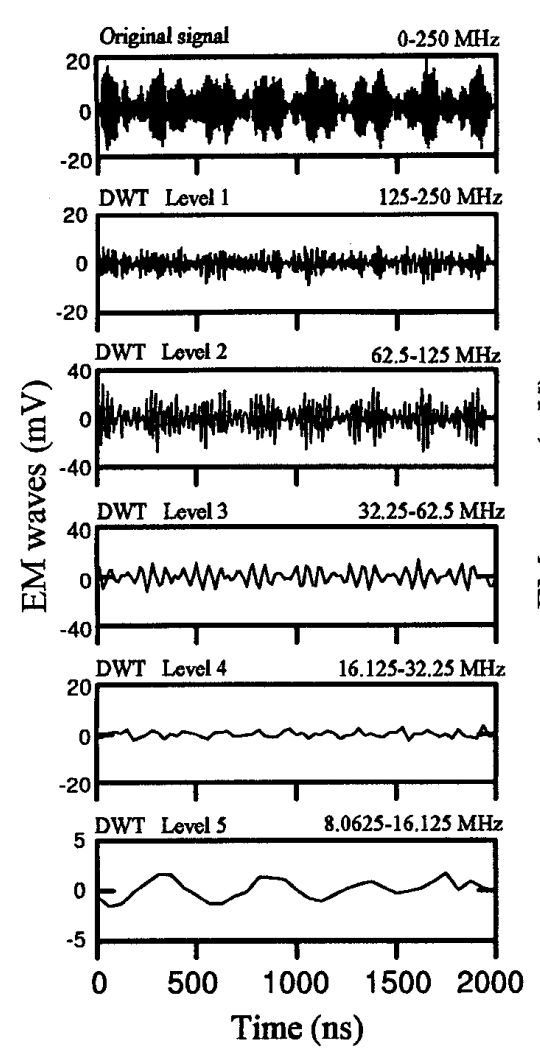

(a) background noises
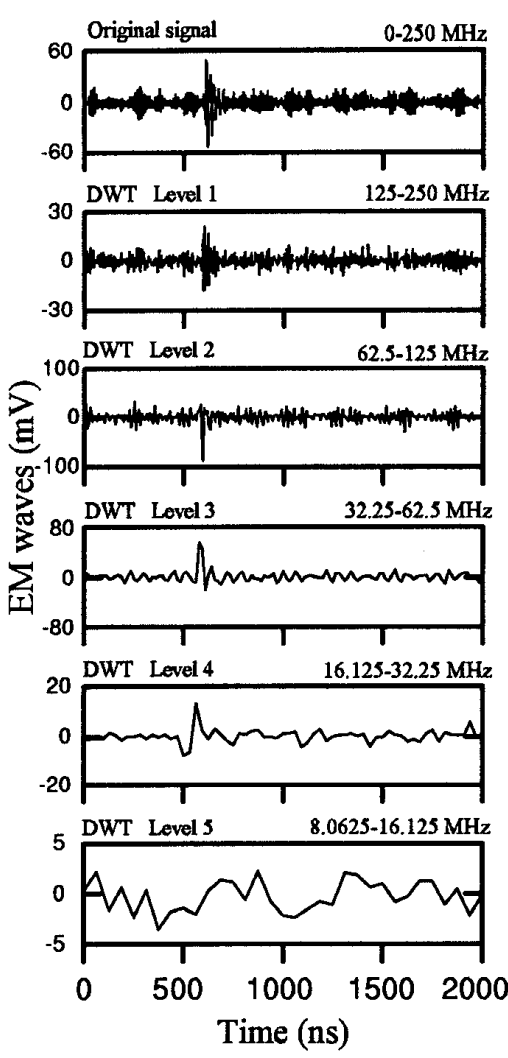

(b) faulty insulators No.1
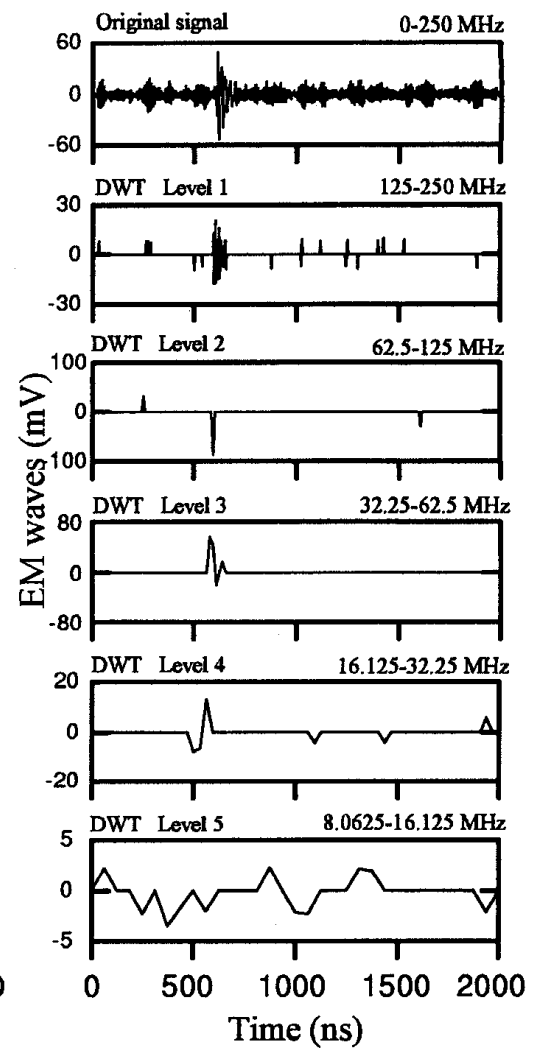

(c) Threshold processing of (b)

Fig. 5. The DWT decomposition of EM waves in 5 Levels

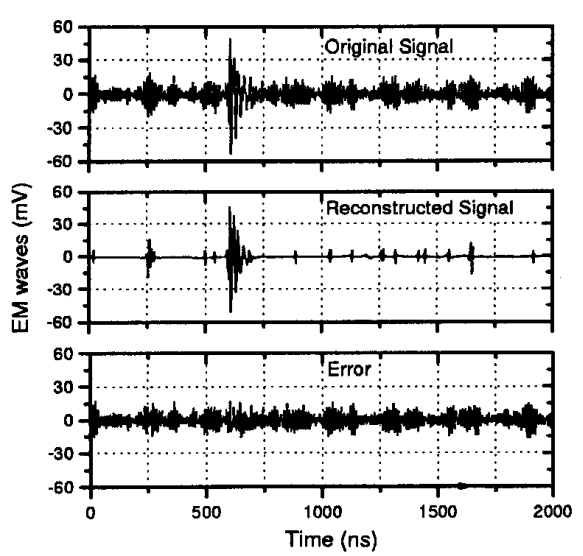

Fig. 6. Comparison of the reconstructed signal with the original one of EM wave in noisy condition

stationary background noises are significantly removed by applying threshold processing on sub-band signals.

To evaluate the proposed method, the spectral analysis by fast Fourier transform and continuous wavelet transform has been applied to both the original PD signal and its reconstructed one. The spectral analysis of reconstructed signal, which is different to that of the original one, become to be similar to that of the other shown example of signals, which is little influenced by noises.

In the future, we plan to monitor and analyze the partial discharge signals in real-time. The automatic classification system will be developed based on the spectral analysis and artificial neural networks.

\section{REFERENCES}

[1] J. Robert Ashley, Are power lines unsafe ?, IEEE Spectrum Magazine, July 2000, Vol.37, No. 7, pp. 21-23.

[2] NHK Training center, The effect of pulsed noise from distribution lines and its measurement, 1996 (in Japanese).

[3] A. Tungkanawanich, Z-I. Kawasaki, J. Abe, and K. Matsu-ura, Location of partial discharge source on distribution line by measuring emitted pulse-train electromagnetic waves, Proceedings of IEEE winter meeting 2000, Singapore, 23-27 January, 2000.

[4] A. Tungkanawanich, Z-I. Kawasaki, K. Matsu-ura, Location of Multiple PD Sources on Distribution Lines by Measuring Emitted Pulse-Train Electromagnetic Waves, to be published in Trans. IEE Japan, Vol. 120-B, No. 11, 2000.

[5] A. Tungkanawanich, Z-I. Kawasaki, K. Matsu-ura, Analysis of VHF-wideband pulse-train electromagnetic noises caused by partial discharge on power distribution lines, Proceedings of Second International Workshop on High Voltage Engineering (IWHV 2000), Totteri, Japan, 1-2 November, 2000.

[6] Charles K. Chui, An introduction to wavelets, Academic Press, Inc. , 1992. 
[7] Oinis Chaari, Michel Meunier, Francoise Brouaye, Wavelet: $A$ new tool for the resonant grounded power distribution systems relaying, IEEE Trans. on Power Delivery, Vol.11, No.3, July 1996, pp. 1301-1308.

[8] R. Polikar, Wavelet Tutorial Part IV: Multiresolution Analysis: The discrete wavelet transform, Publication on internet web at http://www.public.iastate.edu/ rpolikar/WAVELETS/waveletindex.html.

[9] C. Sidney Burrus, R. A. Gopinath, H. Guo, Introduction to wavelets and wavelet transforms: A primer, Prentice-Hall, Inc., 1998.

Ampol Tungkanawanich received a bachelor degree in Electrical Engineering from Chulalongkorn University (Thailand) in 1995. He came to Japan to continue his graduate study under the support of Japanese government (Monbusho scholarship). He received a master degree from Department of Electrical Engineering, Osaka University in 1999 and is currently pursuing a Ph.D. degree there. His research mainly deals with partial discharge, measurement system, fault analysis on power systems, signal processing.

Effrina Yanti Hamid was born in Indonesia. She received the bachelor and master degrees in Telecommunication Engineering from Bandung Institiute of Technology, Indonesia in 1995 and 1998, respectively. Now, she is a Ph. D. student in the Department of Electrical Engineering, Osaka University. Her main interest is in signal processing and its application to power system.

Zen-Ichiro Kawasaki received his B.S., M.S. and Dr. Eng. degrees in Electrical Engineering from Osaka University in 1973, 1975 and 1978, respectively. In 1989, he joined the Department of Electrical Engineering, Osaka University and is currently an associate professor. His research mainly concerns the observation of lightning, and the diagnosis techniques for power equipment. Dr. Kawasaki is also a member of the Society of Atmospheric Electricity of Japan.

Kenji Matsuura received his B.S. and M.S. degrees in Electrical Engineering from Osaka University in 1960 and 1962, respectively. In 1977, he joined the Department of Electrical Engineering, Osaka University and is currently a professor. His research concerns the generation, transmission, and control of electric power. Dr. Matsuura is a member of the Institute of Energy and Resources. 